\title{
A Brief Review of Antifungal Drugs Old and New
}

\author{
Richard W Dudley* \\ Department of Pharmaceutical Sciences, The University of Findlay, USA
}

*Corresponding author: Richard W Dudley, Department of Pharmaceutical Sciences, The University of Findlay, Findlay, OH 45840, USA

Submission: 侮April 3, 2018; Published: 侮 May 24, 2018

\begin{abstract}
Advances in chemotherapy, immunotherapy, and the treatment of HIV have had a tremendous impact on survival significantly decreasing diseaserelated morbidity. However, such therapies have increased the number of patients with a compromised immune system thereby predisposing to opportunistic fungal infections. Despite currently available antifungal drugs, mortality rates remain elevated. Drug approvals for cancer, HIV, and transplantation continue and have significantly outpaced antifungal drug therapy development. Here, a brief review of current antifungal therapy is presented as well as investigational antifungal agents with novel mechanisms of action or molecular targets.
\end{abstract}

Keywords: Anti fungal; Opportunistic infections; Candida spp; Echinocandins; Azole antifungal

\section{Introduction}

Invasive fungal infections caused by opportunistic organisms such as Candida and Aspergillus species are of significant concern as the population of immune compromised patients in society today rises [1-4]. Additionally, increases in the number of Mucorrelated infections is equally or more concerning as effective treatments are somewhat limited [5,6]. The treatment of invasive fungal infections attempts to target metabolic aspects unique to the offending pathogen. Today, two main classes of antifungals drugs are routinely employed to combat invasive fungal infections-azole antifungals and echinocandins. In addition, the polyene antifungal amphotericin-B continues to see clinical use and historically the go to drug for fungal infections. However, amphotericin-B is fraught with adverse effects and carries the potential for renal toxicity [7]. The development of lipid-based formulations of amphotericin-B represented efforts to circumvent toxicity and adverse effects including infusion-related reactions [8]. However, the cost of such formulations is significant. Given today's increasing immune compromised population, the need for cost-effective, therapeutic antifungal agents remains at the forefront of drug development. To combat resistance and increase spectrum of activity, progress has been made in the design and development of newer agents with enhanced activity and potentially a decreased in cost.

\section{Azole antifungals}

The prototypical azole antifungal, ketoconazole, was released to the US market in 1981. This imidazole-bearing agent, and all subsequent azole antifungals, targets the fungal CYP-51 enzyme system which is also known as $14 \alpha$-demethylase $[9,10]$. CYP-51 catalyzes the removal of a methyl group from lanosterol and represents a key enzyme in the synthetic route leading to to the formation of the fungal sterol ergosterol. Inhibition of CYP-51 results in fungal membrane disruptions leading to a fungicidal effect. Ketoconazole has been removed from clinical use for systemic fungal infections due to the risk of hepatic injury, drug-drug interactions, and the risk of decreased human steroid synthesis. Fluconazole, voriconazole, posaconazole, and isavuconazole now represent the current arsenal of azole antifungals.

The triazole ring system for CYP-51 binding. molecular flexibility in a different aspect of these agents allows for varying affinity for CYP-51 in different fungal strains [11]. For example, fluconazole is known to have activity against various Candida species including C. albicans. However, resistance exists or is becoming an issue when using fluconazole to treat C. glabrata, C. tropicalis, and C. krusei [12]. On the other hand, voriconazole is known to possess activity against several fluconazole-resistant Candida strains. Additionally, fluconazole lacks activity against Aspergillus while voriconazole retains use in this infection setting. Isavuconazole, which evolves from administration of the prodrug isavuconazonium sulfate, represents a major advance in azole antifungal therapy. This agent, approved for use in the US in 2015, bears the requisite triazole ring along with a unique side-chain that affords affinity for both Aspergillus CYP-51 as well as the CYP-51 enzyme in molds responsible for causing invasive mucormycosis (eg. Rhizopus, Mucor, Cunninghamella) [13,14]. Posaconazole has been reported to demonstrate utility in the treatment of mucormycosis, however such use is currently offlabel. 


\section{Echinocandins}

Echinocandin B, discovered in 1974, stood to be a significant addition to, at the time, a dearth of antifungal agents in clinical use [15]. This cyclic lipophilic hexapeptide was isolated as a metabolite of Aspergillus nidulans, and later was shown to impair fungal cell wall synthesis. Due to poor solubility and formulation issues as well as severe adverse effects, echinocandin B was not pursued as a clinical agent, However it did inspire the development of semisynthetic analogs including the first-in-class agent caspofungin and latter agents micafungin and anidulafungin [16].

The fungal cell wall is composed of glucan polymers arranged in a 1-3 or a 1-6 linkage with the $\beta(1-3)$-glucan polymer, by weight, being significantly higher in content. The enzyme $\beta(1-$ 3)-glucan synthase is responsible for polymerizing glucan glucan and was shown to be the target of the echinocandins. Through non-competitive inhibition, agents like caspofungin will inhibit the synthase enzyme significantly weakening the fungal cell wall. Lysis and a resulting fungicidal effect ensues. The lack of an equivalent enzyme in humans lends to selective toxicity. Clinically relevant echinocandis are structurally related to echinocandin B. All retain a cyclic hexapeptide core structure and also possess some form of lipophilic tail which lends to anchoring in fungal plasma membranes.

All three currently approved echinocandins have a somewhat limited spectrum of activity and find most clinical use in the treatment of invasive candida infections. In addition to candidemia and invasive candidiasis, caspofungin and micafungin have been used as salvage therapy for invasive aspergillosis as well as for empiric treatment in neutropenic patients [17]. Echinocandins have shown utility in the treatment of azole-resistant candida infections as well. Mutations in the gene encoding the $\beta(1-3)$ glucan synthase (Fks1-Fks2 complex) have been described and are reported to result in resistance [1-3]. Comparatively fewer drug-drug interactions exist for all echinocandins relative to azole antifungals making them attractive agents for candida infections.

\section{Investigational antifungal agents}

Both azole antifungals and echinocandin derivatives have significantly contributed to the treatment of invasive fungal infections. However, fungi will evolve and resistance is expected. The ongoing development of agents which circumvent resistance will be critical as we encounter highly drug-resistant fungal strains such as Candida auris $[18,19]$. Developing novel dosing schemes through enhanced kinetic and dynamic parameters can also have a positive impact on antifungal treatment. To this end, several agents in clinical development are worth noting.

All current echinocandins are administered intravenously on a daily basis. However, a novel derivative, CD-101 (rezafungin), is currently in clinical trials and is regarded as a once-weekly echinocandin [20]. Rezafungin has a similar spectrum of activity as other echinocandins yet is suggested to have a half-life of up to 130 hours after a single $400 \mathrm{mg}$ dose. Such a dosing schedule may expedite patient discharge thereby decreasing healthcare expenditures.

SCY-078 represents a first-in-class oral triterpene inhibitor of $\beta$ (1-3)-glucan synthase and is currently in phase 3 development [21]. It is also being investigated in an open-label study for the emergent treatment of Candida auris infections [22]. SCY-078 represents the first $\beta(1-3)$-glucan synthase inhibitor being developed in both intravenous and oral formulations. Also, while the molecular target may overlap with echinocandins, crossresistance is not expected.

T-2307 is a novel arylamidine in development for the treatment of echinocandin-resistant candida infections. This molecule, structurally similar to the antifungal/antiprotozoal agent pentamidine, induces a collapse in the mitochondrial membrane potential, and has been shown to possess activity against a variety of fungal pathogens including echinocandin-resistant and azoleresistant Candida spp, Cryptococcus neoformans and Aspergillus fumigatus $[23,24]$.

\section{Conclusion}

Antifungal therapy has evolved significantly since the 1960's. Amphotericin-B was the mainstay of treatment for invasive fungal infection for decades until the introduction of the azole antifungals in the 1980's. Subsequent optimization of azoles as well as new formulations of amphotericin-B has greatly improved fungal-related morbidity and mortality. The introduction of echinocandins has contributed to the improvements in outcomes related to invasive fungal infections. Optimization of formulations and pharmacokinetic or pharmacodynamics properties is also improving survival. Despite these advances, invasive fungalrelated mortality remains high and resistance to existing agents is concerning. The identification of both novel agents (SYC-078 and T-2307) and novel fungal targets (mitochondrial membrane potential) is of paramount importance to our successful treatment of fungal infections in particular as the population ages and the number of immune compromised patients rises.

\section{References}

1. Baddley JW (2011) Clinical risk factors for invasive aspergillosis. Med Mycol 49(Suppl 1): S7-S12.

2. Bassetti M, Righi E, Montravers P, Cornely OA (2018) What has changed in the treatment of invasive candidiasis? A look at the past 10 years and ahead. J Antimicrob Chemother 73(suppl 1): i14-i25.

3. Donnelly JP (2013) A multidisciplinary approach to managing invasive fungal disease. Introduction and aims. J Antimicrob Chemother 68(Suppl 3): iii3-iii4.

4. Muskett H, Shahin J, Eyres G, Harvey S, Rowan K, et al. (2011) Risk factors for invasive fungal disease in critically ill adult patients: a systematic review. Crit Care 15(6): R287.

5. Ibrahim AS, Kontoyiannis DP (2013) Update on mucormycosis pathogenesis. Curr Opin Infect Dis 26(6): 508-515.

6. Spellberg B (2017) Mucormycosis pathogenesis: Beyond Rhizopus. Virulence 8(8): 1481-1482.

7. Laborin LR, Vargas CMN (2009) Amphotericin B: side effects and toxicity. Rev Iberoam Micol 26(4): 223-227. 
8. Dix SP, Andriole VT (2000) Lipid formulations of amphotericin B. Curr Clin Top Infect Dis 20: 1-23.

9. Degreef H, Kerckhove MV, Gevers D, Cutsem VJ, Bossche VH, et al. (1981) Ketoconazole (R 41400 ) in the treatment of dermatophyte infections: a clinical, mycological, and morphological study. Int J Dermatol 20(10): 662-669.

10. Graybill JR, Drutz DJ, Murphy AL (1980) Ketoconazole: a major innovation for treatment of fungal disease. Ann Intern Med 93(6): 921923.

11. Richardson K, Cooper K, Marriott MS, Tarbit MH, Troke PF, et al. (1990) Discovery of fluconazole, a novel antifungal agent. Rev Infect Dis 12( Suppl 3): S267-S271.

12. Berkow EL, Lockhart SR (2017) Fluconazole resistance in Candida species: a current perspective. Infect Drug Resist 10: 237-245.

13. Denis J, Ledoux MP, Nivoix Y, Herbrecht R (2018) Isavuconazole: A new broad-spectrum azole. Part 1: In vitro activity. J Mycol Med 28 (1): 8-14.

14. Ledoux MP, Denis J, Nivoix Y, Herbrecht R (2018) Isavuconazole: A new broad-spectrum azole. Part 2: pharmacokinetics and clinical activity. J Mycol Med 28(1): 15-22.

15. Nyfeler R, Keller-Schierlein W (1974) [Metabolites of microorganisms. 143. Echinocandin B, a novel polypeptide-antibiotic from Aspergillus nidulans var. echinulatus: isolation and structural components]. Helv Chim Acta 57(8): 2459-2477

16. Balkovec JM, Black RM, Hammond ML, Heck JV, Zambias RA, et al. (1992) Synthesis, stability, and biological evaluation of water-soluble prodrugs of a new echinocandin lipopeptide. Discovery of a potential clinical agent for the treatment of systemic candidiasis and Pneumocystis carinii pneumonia (PCP). J Med Chem 35(1): 194-198.

17. Balashov SV, Park S, Perlin DS (2006) Assessing resistance to the echinocandin antifungal drug caspofungin in Candida albicans by profiling mutations in FKS1. Antimicrob Agents Chemother 50(6): 20582063
18. Vallabhaneni S, Kallen A, Tsay S, Chow N, Welsh R, et al. (2017) Investigation of the First Seven Reported Cases of Candida auris, a globally emerging invasive, multidrug-resistant fungus-United States, May 2013-August 2016. Am J Transplant 17(1): 296-299.

19. Walia K, Chowdhary A, Ohri VC, Chakrabarti A (2017) Multidrugresistant Candida auris: Need for alert among microbiologists. Indian J Med Microbiol 35(3): 436.

20. James KD, Laudeman CP, Malkar NB, Krishnan R, Polowy K (2017) Structure-activity relationships of a series of echinocandins and the discovery of CD101, a highly stable and soluble echinocandin with distinctive pharmacokinetic properties. Antimicrob Agents Chemother 61(2): e01541-e01516.

21. Lepak AJ, Marchillo K, Andes DR (2015) Pharmacodynamic target evaluation of a novel oral glucan synthase inhibitor, SCY-078 (MK-3118), using an in vivo murine invasive candidiasis model. Antimicrob Agents Chemother 59(2): 1265-1272.

22. Larkin E, Hager C, Chandra J, Mukherjee PK, Retuerto M, et al. (2017) The emerging pathogen candida auris: growth phenotype, virulence factors, activity of antifungals, and effect of SCY-078, a novel glucan synthesis inhibitor, on growth morphology and biofilm formation. Antimicrob Agents Chemother 61(5): e02396-2416.

23. Shibata T, Takahashi T, Yamada E, Kimura A, Nishikawa H, et al. (2012) T-2307 Causes collapse of mitochondrial membrane potential in yeast. Antimicrob Agents Chemother 56(11): 5892-5897.

24. Wiederhold NP, Najvar LK, Fothergill AW, Bocanegra R, Olivo M, et al. (2016) The novel arylamidine T-2307 demonstrates in vitro and in vivo activity against echinocandin-resistant Candida glabrata. J Antimicrob Chemother 71(3): 692-695.
Creative Commons Attribution 4.0 International License

For possible submissions Click Here

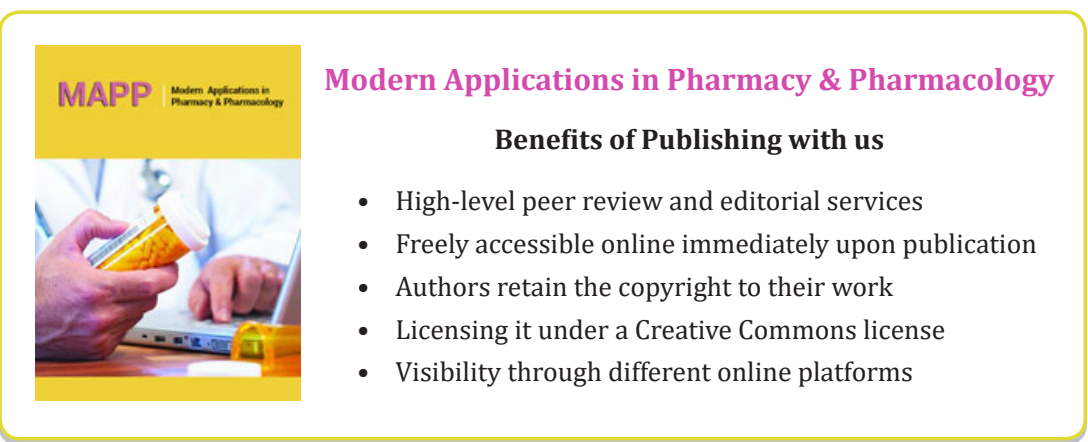

